http://revistas.ucr.ac.cr/index.php/ingenieria

www.ucr.ac.cr / ISSN 1409-2441
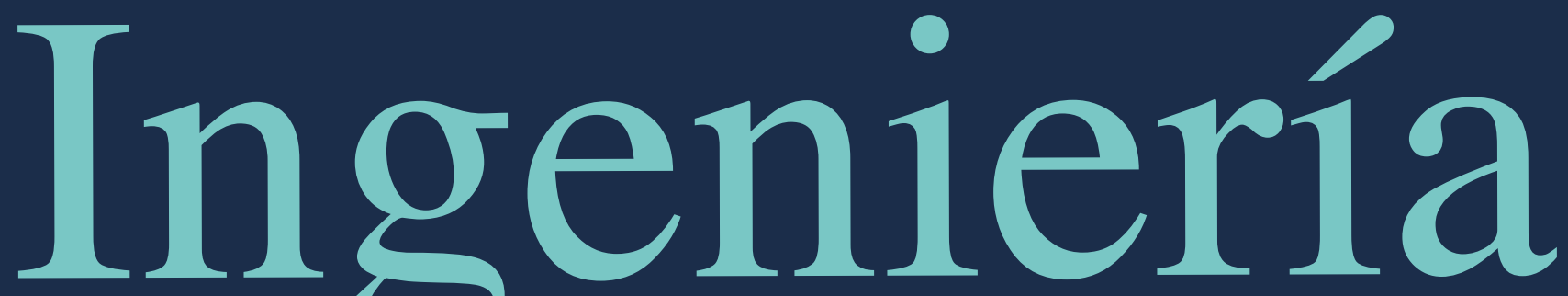

Revista de la Universidad de Costa Rica JULIO / DICIEMBRE 2019 - VOLUMEN 29 (2)

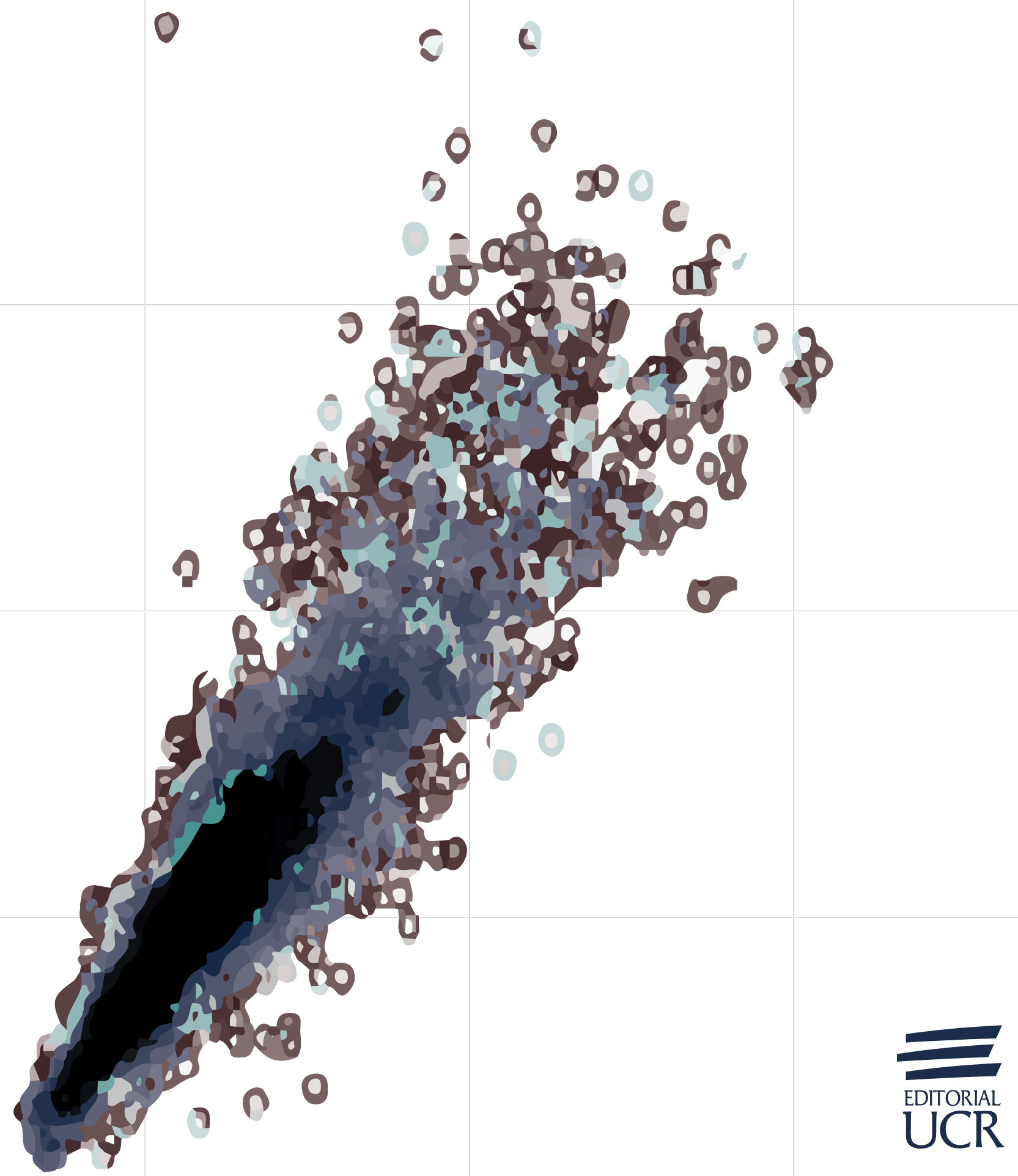




\title{
Evaluando un nodo de reanálisis de oleaje mediante información de una boya colocada en aguas intermedias
}

\author{
Evaluating a wave reanalysis node by a buoy in midwater \\ Henry Alfaro Chavarría, Manuel Corrales González, Georges Govaere Vicarioli, \\ Unidad de Ingeniería Marítima de Ríos y de Estuarios - iMARES, \\ Instituto de Investigaciones en Ingeniería, Universidad de Costa Rica, Costa Rica. \\ Correos: henry.alfaro@ucr.ac.cr, manuel.corralesgonzalez@ucr.ac.cr,georges.govaere@ucr.ac.cr
}

Recibido: 22 de enero 2019

Aceptado: 1 de abril 2019

\section{Resumen}

Las bases de datos de reanálisis de oleaje son una fuente importante de información para desarrollar proyectos de investigación relacionados al clima marítimo y aplicaciones de ingeniería de costas. Esta fuente de información toma todavía más relevancia en lugares donde se carece de registros históricos provenientes de mediciones instrumentales, tal y como sucede en Costa Rica. No obstante, esta información, al tratarse de un producto generado por un modelo numérico, requiere ser calibrada y validada con información instrumental. En este trabajo se ha utilizado la información generada y disponible por la NOAA, de un nodo de reanálisis de oleaje ubicado en aguas profundas frente a la costa del Caribe de Costa Rica, el cual, a su vez, ha sido calibrado y validado con información medida por una boya. El producto que se ha generado es una base de datos de oleaje calibrada desde el 2005 y en continua actualización, la cual podrá utilizarse para distintas aplicaciones en la ingeniería de costas y en aproximaciones de análisis de variabilidad climática del oleaje.

Palabras clave:

Reanálisis, oleaje, boya, clima marítimo, costa Caribe.

\begin{abstract}
The wave reanalysis is an important source of information to develop research projects related to wave climate and coastal engineering applications. This source of information is more relevant in places where it lacks historical registers coming from instrumental measurements as well as happens in Costa Rica. However, as the wave reanalysis is generated from a numerical model, it needs to be calibrated and validated with instrumental measurement. This investigation has used the wave reanalysis's node located in deep water, coming from NOAA, in front of Caribbean's Costa Rica coast. This information has been calibrated and validated with a buoy information. The product is a wave data base calibrated from 2005 onwards, which it is monthly updated; moreover, this information could be used in many coastal engineering applications and in wave climate variability analysis approximations.
\end{abstract}

Keywords:

Reanalysys, wave, buoy, wave climate, Caribbean coast. 


\section{INTRODUCCIÓN}

El oleaje corresponde a una de las variables geofísicas que presentan un papel fundamental en distintos procesos naturales en la zona costera, tales como los procesos de erosión y acumulación litoral, los ecosistemas (Ramos, et al., 2012) y el diseño de estructuras marítimas (Gouldby et al., 2014). Para comprender el comportamiento dinámico del oleaje, como una variable fundamental debido a los efectos directos que induce a lo largo de la costa, es habitual abordar su estudio desde un análisis estadístico que responda a su carácter aleatorio en el espacio y tiempo. De esta forma, para definir adecuadamente el oleaje, se requiere información homogénea en el espacio, continua en el tiempo y cuantitativamente fiable.

Algunas de las fuentes de datos relacionadas con el oleaje que presenta características continuas son las series instrumentales de oleaje. No obstante, estas bases de datos no suelen contar con una extensión espacial suficiente; o bien, presentan un carácter discontinuo en el espacio en el caso de las observaciones satelitales (Izaguirre et al., 2011).

Otras de las fuentes de datos de oleaje son las obtenidas mediante técnicas de aproximación numérica (reanálisis), las cuales han cobrado especial protagonismo a lo largo de las dos últimas décadas (Pérez et al, 2017). Las mismas consisten en series históricas continuas y homogéneas, que complementan las mediciones instrumentales que son de características puntuales.

Por una parte, la información de reanálisis, al ser generada por un modelo de generación y propagación de oleaje, es homogénea espacial y temporalmente; no obstante, al mismo tiempo, estas novedosas bases de datos presentan discrepancias con datos instrumentales en sitios al resguardo de islas y en zonas costeras o litorales, zonas donde el oleaje comienza a experimentar importantes procesos de transformación por efecto de la geometría espacial de fondos marinos, topográficos y batimétricos (Cavaleri et al., 2007). A su vez, y a pesar de la mejora en los modelos numéricos empleados, se han detectado divergencias que obedecen, entre otros, a una mala descripción de los campos de datos de viento y a una insuficiente resolución y cantidad forzamientos del modelo (Feng et al., 2006). Debido a estas razones se realizan trabajos con el propósito de corregir, principalmente, la altura de ola de los datos de reanálisis. Sin embargo, los trabajos de validación y calibración de bases de datos de reanálisis se llevan a cabo a partir de mediciones instrumentales, ya sea proveniente de datos de satélite y/o datos medidos por boyas, con lo cual las validaciones y calibraciones son puntuales y corrigen la serie del parámetro de altura de ola del reanálisis en sitios específicos.

El objetivo de este artículo consiste en evaluar y validar una base de datos de reanálisis de oleaje en aguas profundas, usando datos instrumentales medidos por una boya. Lo anterior busca servir de insumo a los proyectos de ingeniería marítima que se desarrollen en el litoral Caribe de Costa Rica. Para ello se ha organizado el artículo en cinco secciones: en la sección 1 se muestra una introducción, en la sección 2 se describen las fuentes de datos utilizadas; la sección 3 muestra la metodología empleada para calibrar los datos de reanálisis. Los resultados obtenidos y la validación de los mismos se presentan en la Sección 4 y, finalmente, la sección 5 contiene las principales conclusiones. 


\section{DATOS}

Los datos de reanálisis de oleaje utilizados son los provenientes del modelo operacional de generación y propagación Wavewatch III (Tolman, 2002), en su versión 4.15 desarrollado por la NOAA (en adelante se denota como WW3) y abierto al público. Este modelo numérico está entre los denominados modelos de tercera generación y es uno de los más recomendados y ampliamente utilizado en conjunto con el modelo de oleaje WAN (Hasselman et al, 1998) para la generación de datos de oleaje a nivel global y en aguas profundas.

El WW3 es forzado con información de viento, diferencias de temperatura de la superficie del océano y concentración de hielo sobre la superficie del mar. Por su parte, la información de viento proviene del sistema GDAS (Global Data Assimilation Scheme) y se utiliza para forzar el sistema de pronóstico GFS (Global Forecast System). Los campos de viento son transformados a una elevación de $10 \mathrm{~m}$ sobre la superficie y están disponibles en intervalos de cada 3 horas. Asimismo, el modelo WW3 cubre la región del Caribe mediante una malla numérica de $1.25^{\circ}$ x $1.0^{\circ}$. Los datos de salida en toda la malla del modelo son los parámetros de oleaje de altura significante (Hs), período pico (Tp) y dirección principal (dp), con una resolución temporal de cada 3 horas; además, contiene datos desde el 2005 y está en continua actualización. El nodo de la malla del modelo utilizado para este trabajo se localiza en aguas profundas frente a los puertos comerciales y centro poblacional más importante del litoral Caribe de Costa Rica, cuyas coordenadas son 10.5 latitud y -83 longitud.

Para el proceso de validación y calibración se utilizan los datos de oleaje medidos por una boya direccional colocada durante el procesos constructivo de la Terminal de Contenedores de Moín (TCM), adyacente al antiguo puerto de Moín en el Caribe de Costa Rica. La boya estuvo fondeada a $14 \mathrm{~m}$. de profundidad, en las coordenadas $10.026467^{\circ}$ latitud, $-83.105933^{\circ}$ longitud. La boya registró los parámetros de oleaje Hs, período pico (Tp) y dp, desde el año 2015 hasta el 2017 de manera cuasi continua, presentando ciertos huecos, durante dicho período.

La Figura 1 muestra las series temporales de los parámetros Hs, Tp y dp del reanálisis y del instrumento en sus respectivas ubicaciones. Se observa que cada parámetro de oleaje del reanálisis sigue la misma evolución temporal de sus respectivos parámetros instrumentales. En relación con el parámetro Hs, específicamente durante el mes de diciembre del 2016, el modelo simula el efecto del huracán Otto, el cual también es registrado por la boya pero con una intensidad menor. Asimismo, las magnitudes de Hs y dp registradas por la boya durante los meses de junio, agosto y setiembre del 2017, que presentan valores significativamente distintos a los del reanálisis, obedecen a que en esos períodos de tiempo la boya se reubicó en la dársena del puerto, por lo que estos registros no se utilizaron para calibrar ni validar. Respecto a los períodos, se observa una adecuada correlación, incluso para los valores de mayor magnitud. La Figura 1, también muestra, que durante el mes de noviembre del 2016, la boya registra valores de Tp que el modelo no logra simular. Las magnitudes de las direcciones del modelo presentan el mismo comportamiento que las medidas por la boya pero con una menor dispersión. No obstante, se evidencian magnitudes de direcciones provenientes del norte, que difieren con los registros de la boya durante los meses de noviembre 
y diciembre del 2016. Durante estos meses la boya reporta magnitudes de dirección proveniente desde el norte, pero con componente también del este.

Otras de las fuentes de información utilizadas en este trabajo son el reanálisis de viento de alta resolución CFSv2 (Climate Forecast System Version 2) (Saha et al., 2014); este reanálisis es generado por el NCEP (National Center for Enviroment Prediction). Los otros datos utilizados son la marea astronómica, la cual se obtienen del programa TOGA (McPhaden et al., 1998) y la información de batimetría que es tomada de la digitalización de la carta náutica número 28049, denominada aproximación a Puerto Limón y Bahía Moín originadas a partir del levantamiento batimétrico de detalle realizado por la supervisión del proyecto TCM.
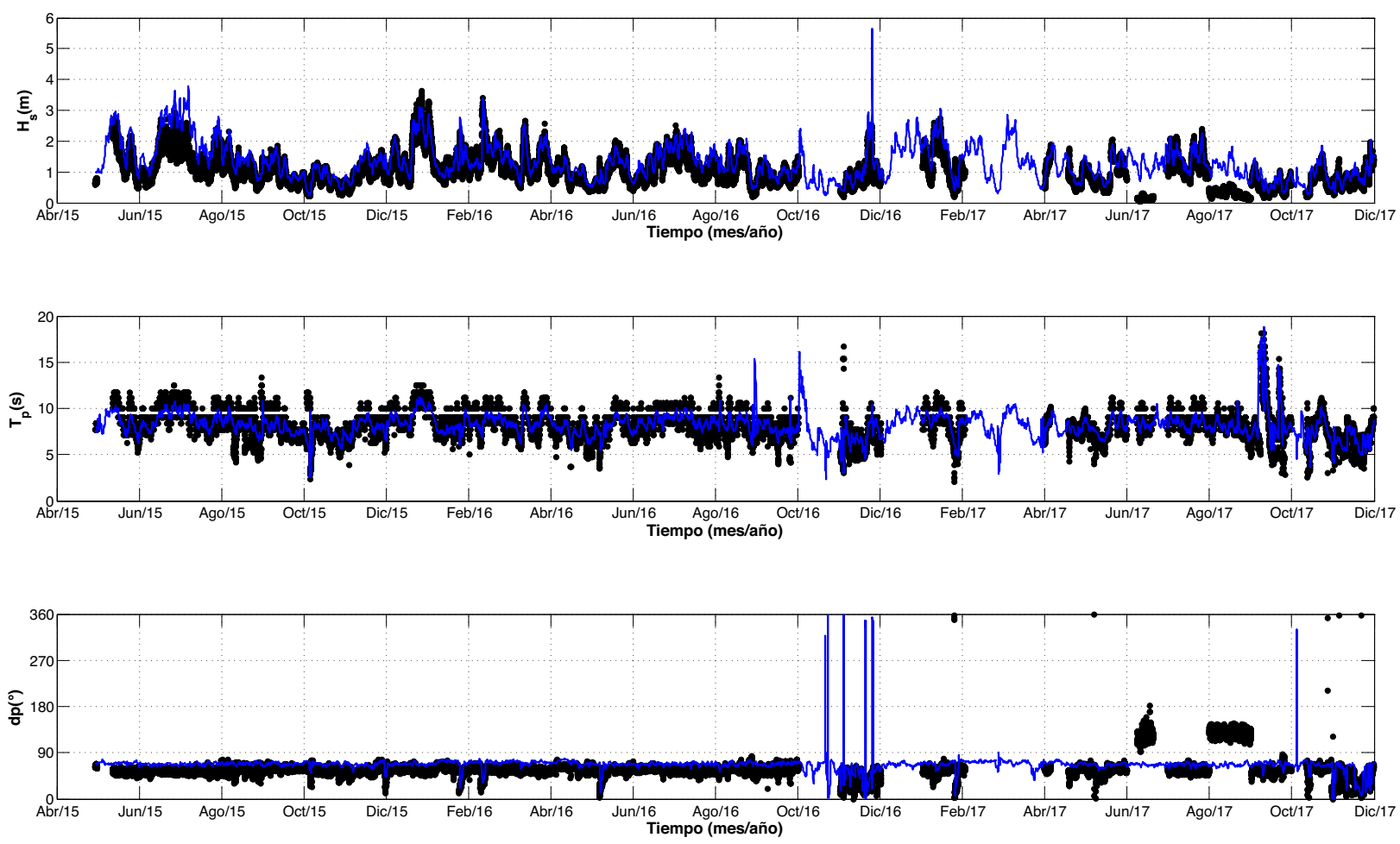

Figura 1. Series temporales de los parámetros Hs, Tp y dp del modelo (azul) y de la boya (negro) para el período de tiempo en que coinciden ambas informaciones.

\section{METODOLOGÍA}

El proceso de calibración, del parámetro Hs del reanálisis de oleaje, es válido para nodos que se localizan en aguas profundas y que se calibran con datos instrumentales ubicados en aguas intermedias, donde los procesos de transformación de oleaje son principalmente la refracción y el asomeramiento. La metodología utilizada se resume a través del esquema que se muestra en la Figura 2.

Se inicia con la selección de los estados de mar del reanálisis de la NOAA que coinciden con el período de medición de la boya. Posteriormente, mediante el modelo de generación y propagación 
de oleaje SWAN (Booij, Ris y Holyhuijsen, 1999), se propagan dichos estados de mar, a los cuales se les conserva las magnitudes de los parámetros Tp y dp. Sin embargo, al parámetro Hs se le sustituye la magnitud por un valor unitario. A su vez, el modelo SWAN es forzado con la información respectiva del reanálisis de viento de alta resolución CFSv2, los datos de la marea astronómica del programa TOGA y la información de batimetría de la carta náutica y del levantamiento batimétrico de detalle.

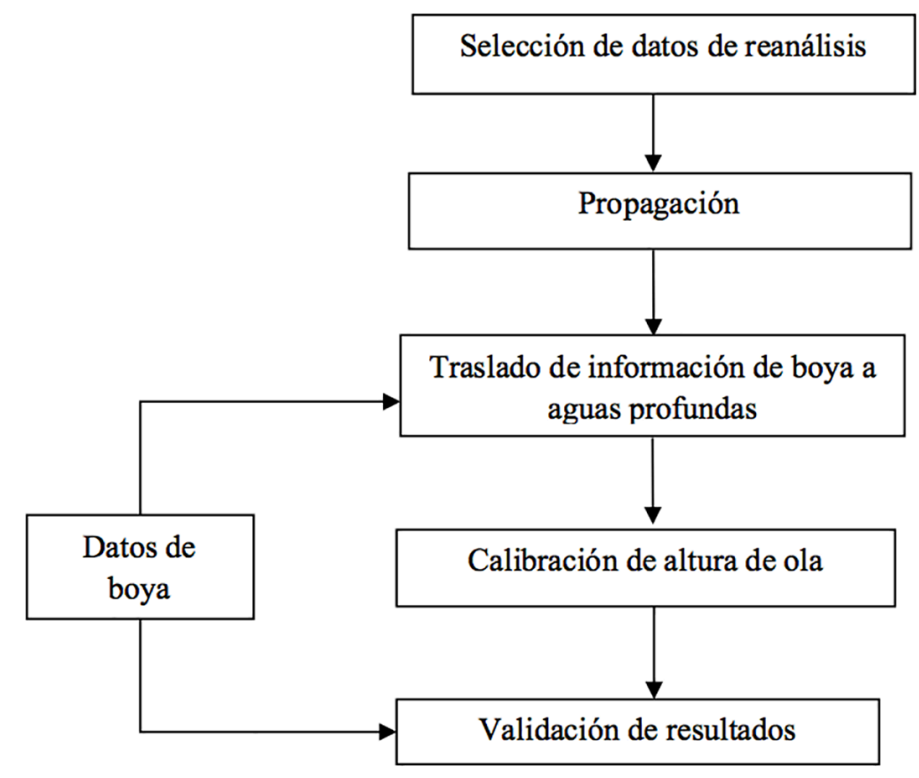

Figura 2. Esquema de la metodología empleada para la calibración del parámetro Hs del reanálisis con datos de boya en aguas intermedias.

Los resultados de propagar cada estado de mar son equivalentes a los coeficientes de propagación, los cuales se utilizan para trasladar el parámetro Hs registrado por la boya, desde su ubicación hasta el sitio del nodo de reanálisis de la NOAA en aguas profundas, para ello se aplica la ecuación 1.

$$
H_{\text {oboya }_{i}}=\frac{H_{\text {sboya }_{i}}}{K_{i}}
$$

donde:

$H_{\text {oboya }}$ : altura de ola significante de la boya en profundidades indefinidas

$H_{\text {sboya }}$ : altura de ola significante registrada por la boya

K: $\quad$ coeficiente de propagación

$i$ : $\quad$ contador que identifica el número del estado de mar

Una vez que la información de la boya se traslada hasta aguas profundas y coincide tanto espacial y temporalmente con la información del reanálisis de oleaje, se comprueba la necesidad de calibrar las magnitudes de Hs del reanálisis de oleaje. Para la calibración del parámetro Hs se sigue la metodología paramétrica dependiente de la dirección del oleaje (Mínguez et al., 2011). El algoritmo matemático utilizado es el siguiente: 


$$
H_{S}^{C}=a^{R}(\theta)\left[H_{S}^{R}\right]^{b^{R}(\theta)}
$$

donde:

$H_{s}^{R}$ : es la altura de ola significante del reanálisis de la NOAA

$H_{s}^{C}$ : es la altura de ola significante calibrada

$a^{R}(\theta)$ y $b^{R}(\theta)$ : son los parámetros que dependen de la dirección media del oleaje de la serie del reanálisis de la NOAA

Tras llevar a cabo la calibración, la ecuación 2 se aplica al resto de la totalidad de los datos del parámetro Hs de la serie de reanálisis de oleaje.

Para comprobar la calidad de la calibración se utilizan gráficos de dispersión (scatter plot), en conjunto con la recta bisectriz y los descriptores de calidad de ajuste como son el sesgo o BIAS, la raíz del error cuadrático medio (RMSE, por sus siglas en inglés), el coeficiente de correlación de Pearson $(\rho)$ y el índice de dispersión (SI). Estos descriptores de ajuste se definen por medio de las siguientes ecuaciones:

- Sesgo (BIAS): es la definición sistemática entre dos variables, mide la diferencia entre ambas variables y se define como:

$$
B I A S=\bar{x}-\bar{y}
$$

- Raíz del error cuadrático medio (RMSE): mide la exactitud con la que se parecen dos variables, se define como:

$$
\text { RMSE }=\sqrt{\frac{1}{n} \sum_{i=1}^{n}\left(x_{i}-y_{i}\right)^{2}}
$$

- Coeficiente de correlación de Pearson $(\rho)$. Mide la intensidad de la relación de igualdad entre dos variables. Está definido entre -1 y 1, cuando existe correlación lineal perfecta entre las dos variables (los datos de $\mathrm{X}$ e Y son iguales) $\rho=1$ si la correlación es lineal y positiva o $\rho=-1$ si la correlación es lineal y negativa, si $\rho=0$ no hay correlación lineal entre las variables.

$$
\rho=\frac{\sum_{i=1}^{n}\left(x_{i}-\bar{x}\right)\left(y_{i}-\bar{y}\right)}{\sqrt{\sum_{i=1}^{n}\left(x_{i}-\bar{x}\right)^{2}} \sqrt{\sum_{i=1}^{n}\left(y_{i}-\bar{y}\right)^{2}}}
$$

- Índice de dispersión respecto a la recta bisectriz; si todos los puntos se fijan sobre la recta bisectriz, el parámetro adimensional SI toma el valor de cero: 


$$
S I=\frac{R M S E}{\bar{x}}
$$

donde $X$ son los datos de referencia $\left(x_{1}, x_{2}, \ldots, x_{i}, \ldots, x_{n}\right)$ (datos instrumentales) y $Y\left(y_{1}, y_{2}, \ldots, y_{i}, \ldots, y_{n}\right)$ los datos a evaluar o aproximaciones (reanálisis).

Por último, para validar los resultados obtenidos se trasladan de nuevo, mediante los coeficientes de propagación conseguidos a partir de la ecuación 1, los estados de mar calibrados que tiene correlación temporal con los datos instrumentales desde aguas profundas hasta el sitio de la boya Para comprobar la calidad de los resultados de la validación se utilizan nuevamente los descriptores de calidad de ajuste.

\section{RESULTADOS}

La Figura 3 muestra, por medio del gráfico de serie temporal del parámetro Hs, el resultado de trasladar la información de la boya hasta profundidades indefinidas luego de aplicar la ecuación 1. Es evidente que ambas series presentan el mismo comportamiento en el tiempo y sus magnitudes son similares. Sin embargo, existen algunos eventos extremos de Hs del reanálisis que sobreestima lo que sucede en la realidad, tal y como se muestra en ciertos días del mes de julio 2015 y en noviembre del 2016, durante el evento meteorológico correspondiente al huracán Otto.

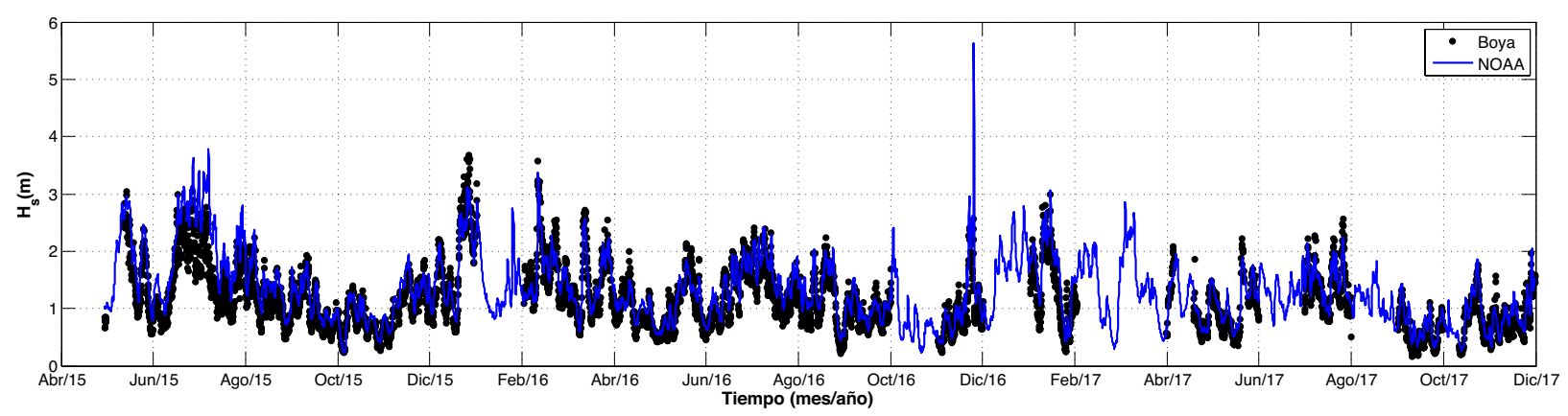

Figura 3. Series temporales de Hs de la boya en profundidades inde nidas e información del reanálisis sin calibrar.

La Figura 4 muestra los resultados de calibrar el parámetro Hs siguiendo la metodología de (Mínguez et al., 2011) y aplicando la ecuación 2. Se observa cómo, en comparación con la Figura 3, las magnitudes de Hs de la serie del reanálisis se ajustan más a los valores de referencia, esto se hace evidente en relación con los eventos extremos del reanálisis de oleaje. 


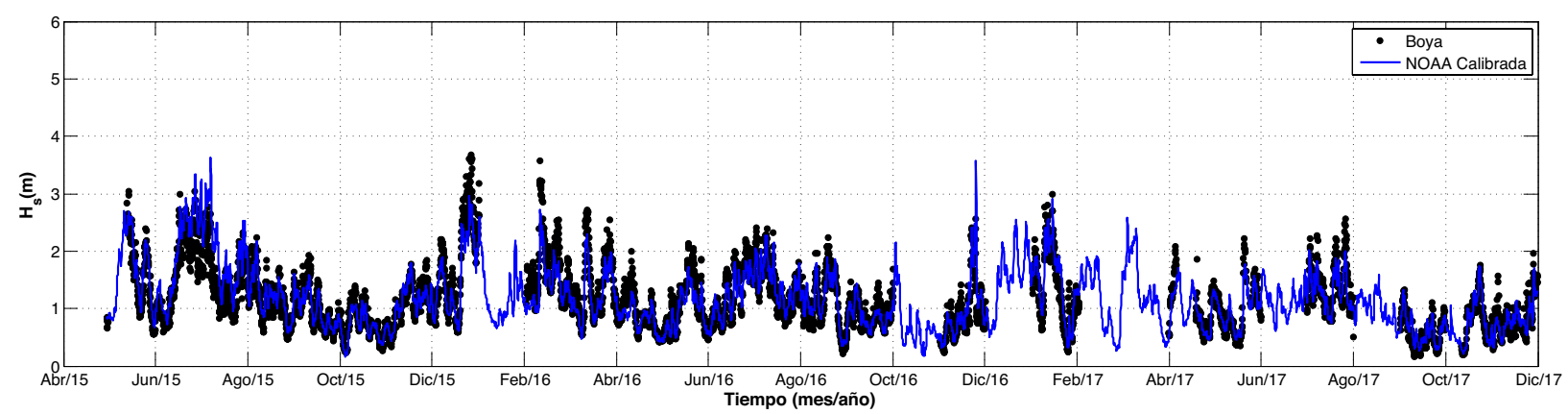

Figura 4. Series temporales de Hs de la boya en profundidades inde nidas e información del reanálisis calibrada.
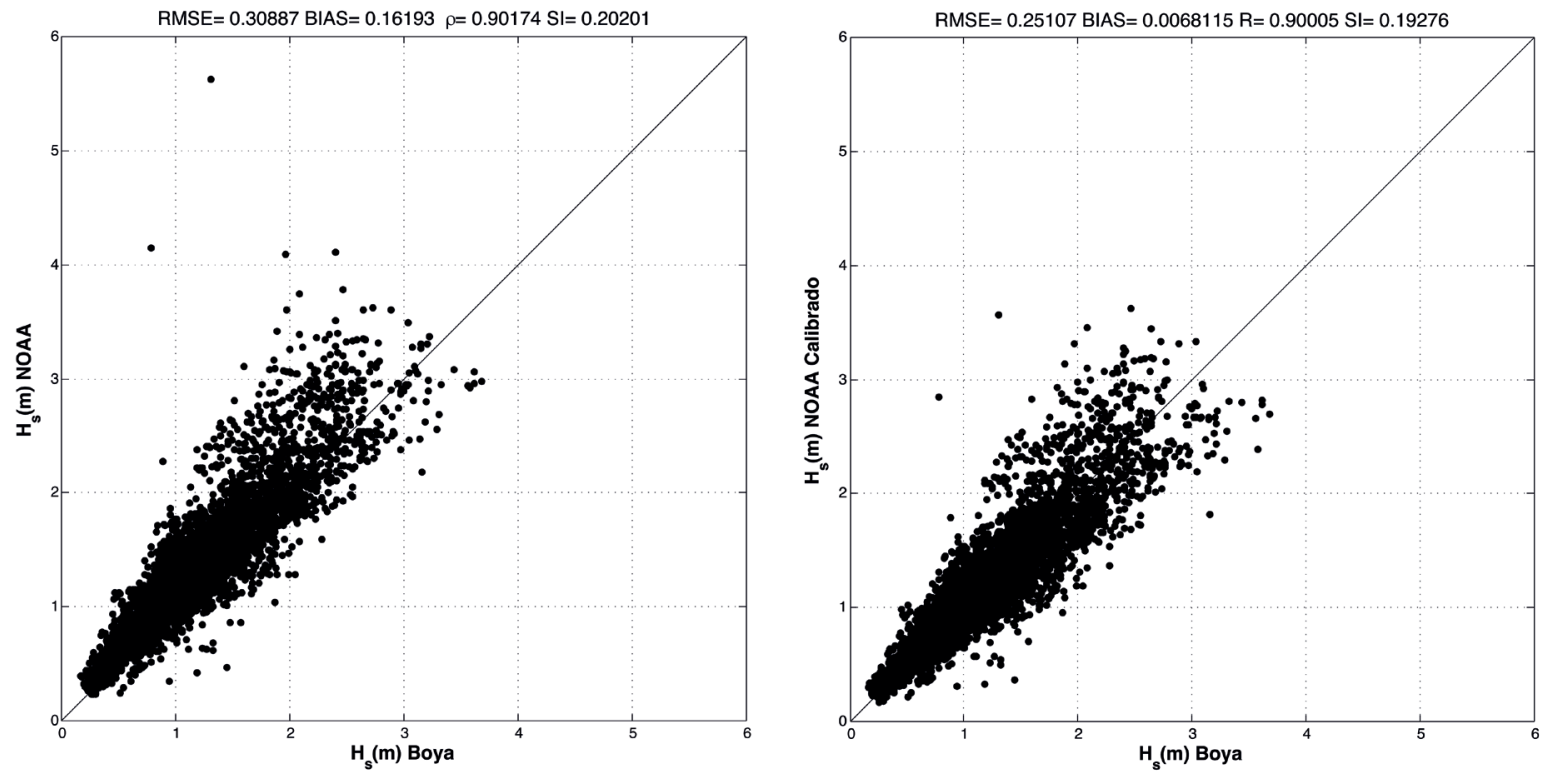

Figura 5. Gráficos scatter plot en conjunto con los parámetros de calidad de ajuste. A la izquierda serie de reanálisis sin calibrar y a la derecha serie de reanálisis calibrada.

Para comprobar la calidad de los resultados de la calibración, la Figura 5 muestra los gráficos scatter plot antes y después de calibrar, en conjunto con los descriptores de calidad de ajuste. La calibración ha disminuido las discrepancias entre el reanálisis de la NOAA y la serie medida por el instrumento. Asimismo, se incluyen los descriptores estadísticos de ajuste (BIAS, RMSE, $\rho$ y SI), tanto para la serie sin calibrar así como posterior a la calibración. Los valores de dichos parámetros verifican los buenos resultados luego de realizar el procedimiento de calibración. El RMSE ha disminuido aproximadamente en $5 \mathrm{~cm}$, la correlación $(\rho)$ y el SI mantienen sus valores los cuales son del orden del $90 \%$ y 0.19 respectivamente, lo que comprueba una alta correlación y una baja dispersión de los datos, y principalmente el parámetro que se mejora es el BIAS, el cual antes de la calibración evidenciaba que los valores estimados por el reanálisis estaban sobrestimados, pero luego de la calibración se mejora esta situación al disminuir el BIAS 16,19cm a 0,70cm. 

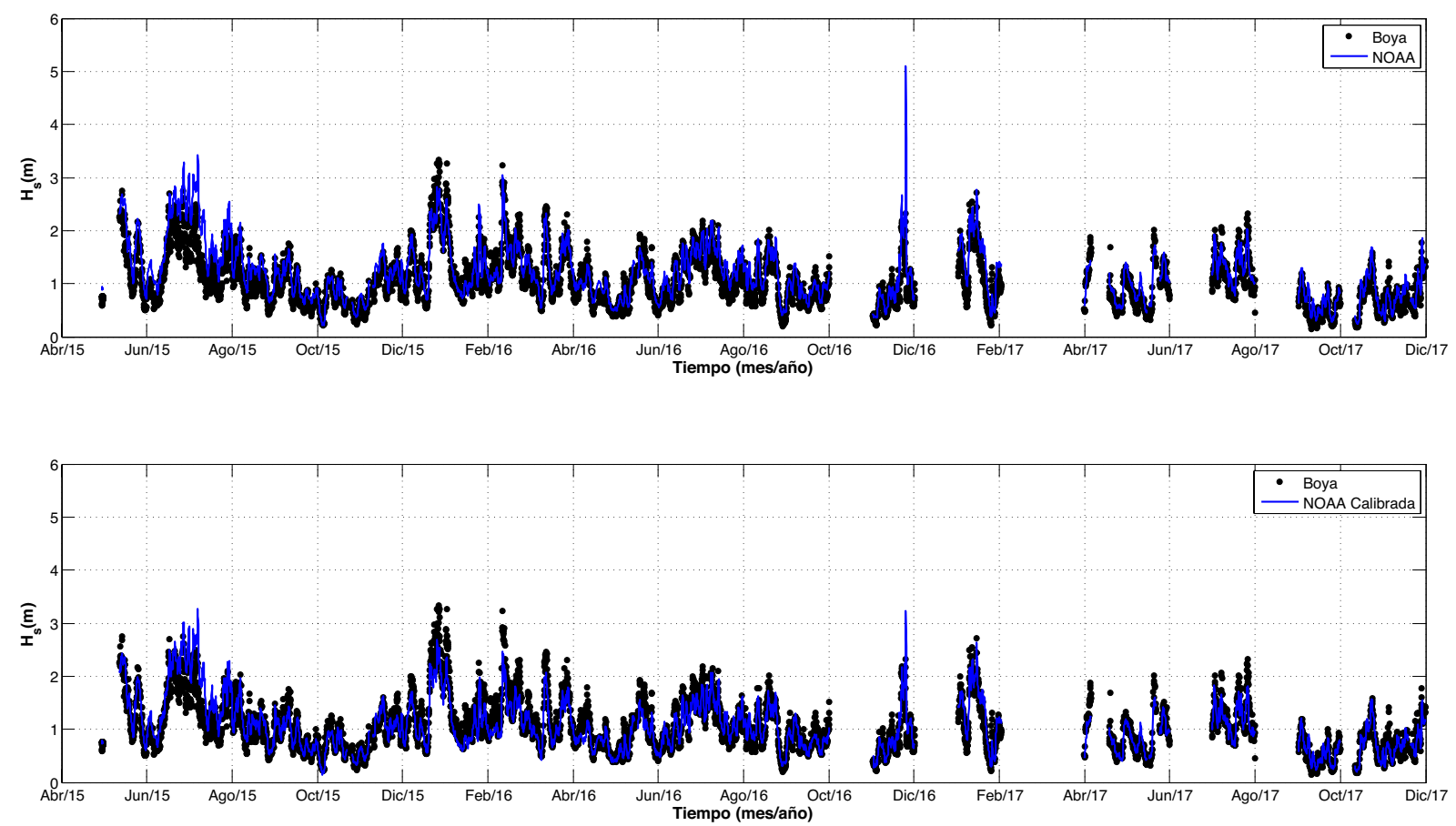

Figura 6. Series temporales de Hs en sitio de la boya. Arriba parámetro Hs de reanálisis sin calibrar y boya, abajo parámetro Hs de reanálisis calibrado y boya.
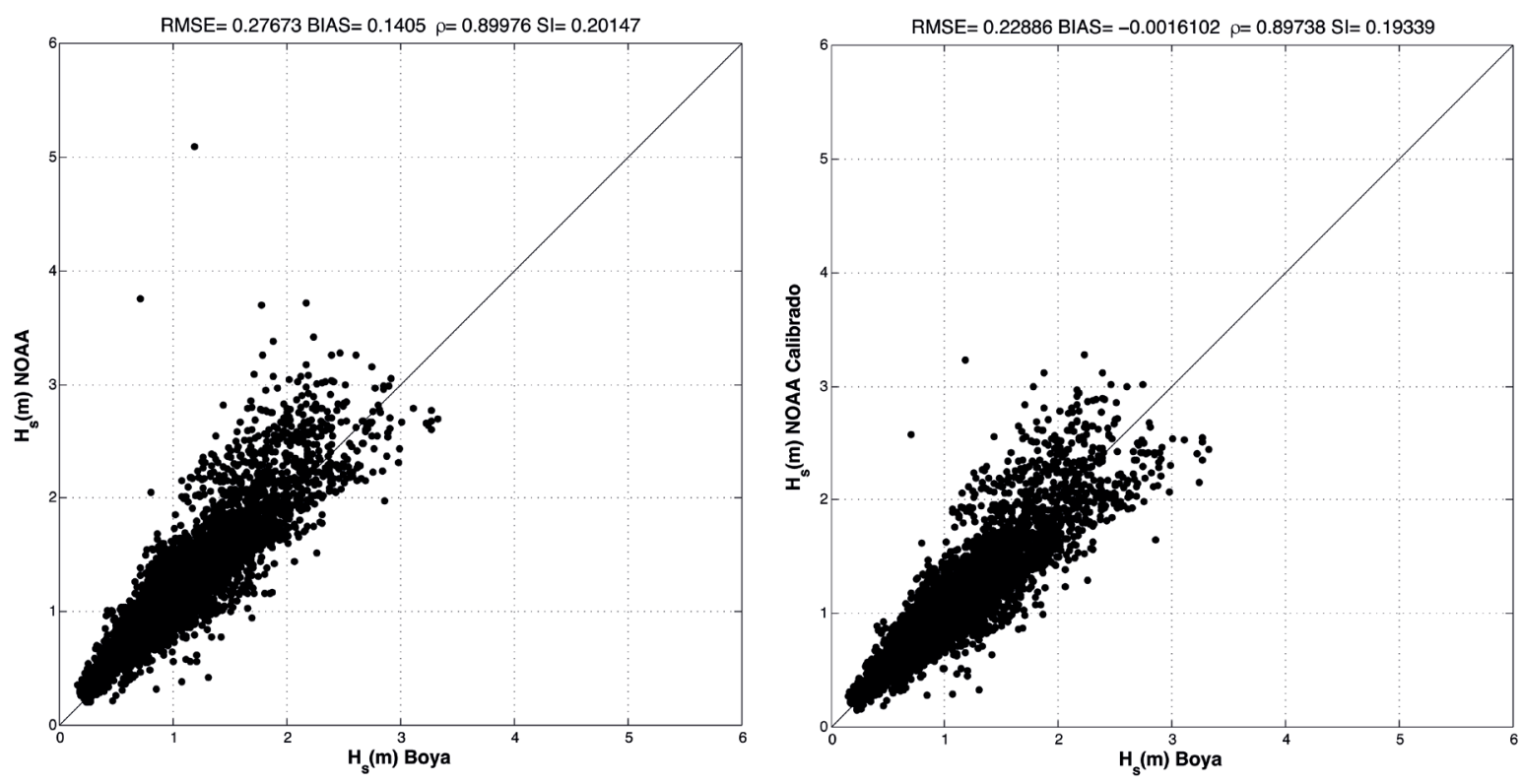

Figura 7. Gráficos scatter plot en conjunto con los parámetros de calidad de ajuste. A la izquierda serie de reanálisis en sitio de medición de la boya sin calibrar y a la derecha serie de reanálisis calibrada.

La Figura 6 muestra los resultados del proceso de validación, para ello se apoya en las series del parámetro Hs de la serie de reanálisis calibrada y sin calibrar, ambas en conjunto con los datos registrados por la boya en el sitio donde ésta última estuvo colocada. Se observa, nuevamente, cómo 
la serie de reanálisis sigue el mismo comportamiento en el tiempo que los datos medidos por la boya, pero la serie de reanálisis sin calibrar presenta algunos eventos de magnitudes mayores a los registrados por la boya, pero una vez calibrado el parámetros Hs estas inconsistencias se reducen, tal y como se muestra la Figura 6.

La Figura 7 muestra los gráficos scatter plot de la validación en conjunto con los descriptores de calidad de ajuste, los cuales evidencian una mejora en las magnitudes de dichos parámetros, lo que valida los resultados del procedimiento aplicado. Como se puede observar, el RMSE ha disminuido en aproximadamente $7 \mathrm{~cm}$, el $\rho$ y el SI presentan valores de $0.89 \%$ y 0.19 respectivamente, lo que comprueba una alta correlación y una baja dispersión de los datos respecto a la mediatriz. El BIAS presenta valores en el orden de los milímetros pasando de 14,05 cm a $-0,16 \mathrm{~cm}$, lo que evidencia que los resultados no muestran un sesgo a valores sobre o subestimados.

\section{CONCLUSIONES}

Se sabe que las bases de datos de reanálisis de oleaje presentan disparidad en relación con lo que realmente sucede en la naturaleza. No obstante, debido a sus características de homogeneidad y continuidad espacio temporal, corresponden a fuentes de información altamente relevantes, principalmente en sitios donde se carece de datos instrumentales como es el caso de muchos países de Latinoamérica. Esta característica de inexactitud suele deberse principalmente a las propias limitantes matemáticas de las herramientas numéricas empleadas, generalmente confeccionadas para ser fiables hasta el límite de aguas indefinidas y sin resolver los clásicos procesos de refracción, asomeramiento, difracción y rotura del oleaje en zonas más someras y en la costa.

Se ha calibrado y validado la información de un nodo de reanálisis de oleaje de la NOAA en aguas profundas frente al Caribe de Costa Rica, con lo cual se ha generado una base de datos homogénea, robusta para realizar climatología y con la capacidad de ser utilizada en trabajos de regionalización del oleaje en la costa del Caribe de Costa Rica. Para llegar a este producto se ha seguido un protocolo, donde se combinan técnicas numéricas y matemáticas que se esquematizan en la Figura 2, pasando por una calibración inicial de la base de datos de reanálisis de oleaje de la NOAA con datos instrumentales, su propagación y su subsiguiente validación.

El producto generado corresponde a una base de datos de oleaje calibrada tres horaria desde el año 2005 y en continua actualización, la cual puede ser explotada para proyectos de ingeniería de costas y puertos que se realicen en la zona litoral del Caribe, principalmente frente a la ciudad de Limón y sus principales terminales portuarias.

Los resultados obtenidos se han evaluado mediante parámetros estadísticos que miden la calidad del ajuste como son la raíz del error cuadrático medio, el sesgo, el coeficiente de correlación de Pearson $(\rho)$ y el índice de dispersión. Los resultados demuestran una mejora en la calidad de los datos del reanálisis de oleaje de la NOAA y por ende la necesidad de calibrar esta base de datos para la zona de estudio. 


\section{AGRADECIMIENTOS}

Se agradece a la NOAA por facilitar los datos del reanálisis de oleaje (http://polar.ncep.noaa.gov/ waves/hindcasts/), al Consejo Nacional de Concesiones y a la empresa APM Terminals por facilitar los datos medidos por la boya durante la construcción de la Terminal de Contenedores de Moín.

\section{REFERENCIAS}

Booij, N., Ris, R. C., y Holthuijsen, L. H. (1999). A third-generation wave model for coastal regions: 1. Model description and validation. Journal of Geophysical Research: Oceans, 104(C4), 7649-7666. https://doi. org/10.1029/98JC02622

Cavaleri, L., Alves, J., Ardhuin, F., Babanin, A., Banner, M., Belibassakis, K., ... y Young, I. (2007). Wavemodelling. The state of the art. Progress in Oceanography, 75(4), $603-674$.

Feng, H., Vandermark, D., Quilfen, Y., Chapron, B., y Beckley, B. (2006). Assessment of wind-forcing impact on a global wind-wave model using the TOPEX altimeter. Ocean Engineering, 3(11-12), 1431-1461.

Gouldby, B., Méndez, F. J., Guanche, Y., Rueda, A., y Mínguez, R. (2014). A methodology for deriving extreme nearshore sea conditions for structural design and flood risk analysis. Coast. Eng. 88, 15-25. https://doi.org/10.1016/j.coastaleng.2014.01.012.

Hasselman, S., Hasselman, K., Janssen, P. A. E. M., Komen, G.T., Bertotti, L., ... y Ewing, J. A. (1998). The WAM model: a third generation ocean wave prediction model. Journal of Physical Oceanography, 18(12), 1775-1810.

Izaguirre, C., Méndez, F. J., Menéndez, M., Losada, I. J. (2011). Global extreme wave height variability based on satellite data, Geophys. Res. Lett. 38(10). https://doi.org/10.1029/2011GL047302.

McPhaden, M. J., Busalacchi, A. J., Cheney, R., Donguy, J.-R., Gage, K. S., Halpern, D., y Takeuchi, K. (1998). The Tropical Ocean-Global Atmosphere observing system: A decade of progress. Journal of Geophysical Research, 103(C7), 14169. https://doi.org/10.1029/97JC02906.

Mínguez, R., Espejo, A., Tomás, A., Méndez, F. J., y Losada, I. J. (2011). Directional calibration of wave reanalysis databases using instrumental data. Journal of Atmospheric and Oceanic Technology, 28(11), 1466-1485. https://doi.org/10.1175/JTECH-D-11-00008.1

Pérez, J., Menéndez, M., y Losada, I. J. (2017). GOW2: A global wave hindcast for coastal applications. Coastal Engineering, 124. Recuperado de http://doi.org/10.1016/j.coastaleng.2017.03.005

Ramos, E., et al. (2012). Coastal waters classification based on physical attributes along the NE Atlantic region. An approach for rocky macroalgae potential distribution, Estuar. Coast. Shelf Sci. 112, 105-114. http://dx.doi.org/10.1016/j.ecss.2011.11.041.

Saha, S., Moorthi, S., Wu, X., Wang, J., Nadiga, S., Tripp, P. y Becker, E. (2014). The NCEP climate forecast system version 2. Journal of Climate, 27(6), 2185-2208. https://doi.org/10.1175/JCLI-D-12-00823.1

Tolman, H. (2002). User manual and system documentation of wavewatch-iii version 2.22. September NOAA/NWS/NCEP Technical Note. 